\title{
Autologous Bi-epitope BCMA-targeted CAR T-cells JNJ-68284528
}

National Cancer Institute

\section{Source}

National Cancer Institute. Autologous Bi-epitope BCMA-targeted CAR T-cells /NL-

68284528. NCl Thesaurus. Code C148498.

A preparation of autologous T-lymphocytes that are transduced, ex vivo, with LCARB38M, a lentiviral vector expressing a chimeric antigen receptor (CAR) containing two bispecific anti-B-cell maturation antigen (BCMA) variable fragments of llama heavy-chain murine antibodies fused to the signaling domain of 4-1BB (CD137), with potential immunostimulating and antineoplastic activities. The antigen-binding region of the CAR is a non-scFv structure targ eting two distinct regions of BCMA. Upon intravenous administration back into the patient, the autologous bi-epitope BCMA-targ eted CAR Tcells JNJ-68284528 are directed to cells expressing BCMA, bind to two different epitopes on BCMA and induce selective toxicity in BCMA-expressing tumor cells. BCMA, a tumorassociated antigen (TAA) and a receptor for both a proliferation-inducing ligand (APRIL) and $\mathrm{B}$-cell activating factor (BAFF), is a member of the tumor necrosis factor receptor superfamily (TNFRSF) and plays a key role in plasma cell survival. BCMA is overexpressed on malignant plasma cells. 